Recepción: 20 / 10 / 2016

Aceptación: 06 / 02 / 2017

Publicación: 29 / 04 / 2017

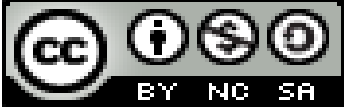

Ciencias de la educación

Artículo de investigación

\title{
Psicología de la educación y la tecnología de la información y la comunicación
}

\author{
Psychology of education and information and communication technology
}

Psicologia educacional e tecnologia da informação e comunicação

Pedro J. Tenorio-Maldonado

pedritojoffre@hotmail.es

Correspondencia: pedritojoffre@ hotmail.es

Magister en Docencia Mención Gestión en Desarrollo del Currículo, Licenciado En Ciencias de la Educación Especialidad Psicología y Orientación Vocacional, Docente de la Universidad Técnica Luis Vargas Torres de Esmeraldas, Esmeraldas, Ecuador. 


\section{Resumen}

En la sociedad, es común escuchar como idea futurista que viviremos en una comunidad del conocimiento donde la educación es una prioridad, por ser el resultado de un complejo proceso de la actividad humana, basado en la elaboración de un conjunto de normas, símbolos, leyes y valores, aceptados por sus miembros. Mediante una revisión bibliográfica fue posible penetrar en el tema de la psicología de la educación y la tecnología de la información y la comunicación, en aspectos como definición de la psicología de la educación, la usabilidad de TIC, la práctica educativa que incorporan las TIC. Se exponen algunos resultados de investigaciones relacionadas con la temática.

Palabras clave: educación; psicología; tecnología de la información; comunicación.

\section{Abstract}

In society, it is common to hear as a futurist idea that we will live in a knowledge community where education is a priority because it is the result of a complex process of human activity, based on the elaboration of a set of rules, symbols, laws And values, accepted by its members. Through a bibliographical review it was possible to penetrate the subject of the psychology of education and information and communication technology, in aspects such as definition of the psychology of education, the usability of ICT, and the educational practice that incorporate ICT. Some results of research related to the subject are presented.

Keywords: education; psychology; information technology; communication.

\section{Resumo}

$\mathrm{Na}$ sociedade, é comum ouvir idéia tão futurista ao vivo em uma comunidade de conhecimento onde a educação é uma prioridade, como o resultado de um processo complexo de atividade humana, com base no desenvolvimento de um conjunto de regras, símbolos, leis e os valores aceitos pelos seus membros. Através de uma revisão da literatura foi possível penetrar o tema da psicologia educacional e tecnologia da informação e comunicação em áreas como a definição de psicologia educacional, usabilidade das TIC prática educativa integração das TIC. Alguns resultados de pesquisas relacionadas ao assunto exposto.

Palavras chave: educação; psicologia; tecnologia da Informação; comunicação. 


\section{Introducción}

Durante todo el tiempo que la psicología educativa ha existido (aproximadamente 90 años), se han originado debates en cuanto a lo que es realmente esta disciplina. Algunas personas consideran que la psicología educativa, son solo conocimientos obtenidos de la psicología y aplicados en las actividades el salón de clases. Otros creen que implica el uso de las técnicas de la psicología para estudiar el salón de clases y la vida escolar. (Woolfolk A. 2010).

La perspectiva que por lo general se acepta actualmente es que la psicología educativa es una disciplina distinta con sus propias teorías, métodos, problemas y técnicas de investigación, su principal objetivo consiste en la comprensión y el mejoramiento de la educación. (Woolfolk A. 2010).

Coll C (2001), plantea que la evolución de la especie humana ha estado asociada, desde sus orígenes, a la creación de artefactos técnicos con el fin de ampliar y extender la capacidad de las personas para actuar sobre la realidad y transformarla, trascendiendo así las limitaciones derivadas de sus características corporales y mentales. Entre todas las tecnologías creadas por los seres humanos, las relacionadas con la capacidad para representar y transmitir la información, tienen especial importancia en la medida en que afectan directamente todos los ámbitos de la actividad de las personas, desde las formas y prácticas de organización social, hasta la manera de comprender el mundo, organizar esta comprensión y transmitirla a otras personas.

Sobre la base de las consideraciones anteriores la importancia del aprendizaje a lo largo de la vida, la aparición de nuevas necesidades formativas, la ubicuidad de las TIC, la necesidad de adquirir competencias estrechamente vinculadas a nuevos espacios personales e institucionales son, todos ellos, factores estrechamente relacionados con la transformación de los espacios educativos tradicionales a los que estamos asistiendo en la actualidad, así como con la aparición de otros nuevos. Las instituciones de educación formal (escuelas, institutos, centros de educación superior, universidades, etcétera) van transformándose progresivamente como consecuencia del impacto de estos factores. (Coll C. 2001).

Las nuevas tecnologías de comunicación e información (en adelante TICs) son esenciales en el aprendizaje, ya que nuestra sociedad hoy, y con toda seguridad en el futuro, estará invadida por éstas. Si consideramos que el conocimiento registrado se duplica cada cinco años, proyectándose 
al 2020 un conocimiento duplicado cada setenta y tres días, ciertamente se impone a nuestros jóvenes una realidad que implica cambios y adaptación, una preparación que sea «life long learning» sólo será posible con las nuevas tecnologías (Brunner, 2002). Por esta razón es fundamental incorporar las TICs al contexto escolar en los talleres y laboratorios de computación, así como también, en toda actividad intra y extraaula, con los docentes, los padres y desde ellos mismos como estrategia de autoaprendizaje. (Peña I S et al. 2006).

En la actualidad con los avances de la tecnología el docente experimenta cambios en su estructura cognitiva en virtud de enfrentar nuevas formas de "aprender para enseñar", es decir, conocer las maneras de tener acceso y aprender a utilizar los diferentes recursos tecnológicos como herramientas para el proceso enseñanza-aprendizaje, dichos constructos derivados del latín, “enseñanza” cuyo significado es señalar, y el aprendizaje entendido como "atrapar”, conceptos empleados para hacer referencia al maestro quien indica el camino para que los estudiantes se apropien del conocimiento, por tanto, la usabilidad de TIC durante la formación inicial docente permitirá conocer la diversidad de recursos tecnológicos que podrían incorporar con fundamento pedagógico en la práctica educativa.( Colorado-Aguilar B, Navarro R. E. 2012).

\section{La usabilidad de TIC}

La usabilidad de TIC, es un aspecto que hace referencia al uso accesible y tipo de experiencia de uso cuando existe interacción entre sistemas o dispositivos tecnológicos con el hombre. (Colorado-Aguilar B, Navarro R. E. 2012).

En el ámbito educativo la usabilidad representa la adecuada apropiación y accesibilidad que se genera al momento de interactuar con el recurso tecnológico, y cuando el docente se pregunta ¿qué recursos existen y puedo utilizar en mi labor educativa?, ¿cómo empleo las TIC dentro de mi planeación didáctica y a la hora de realizar mi clase?, ¿existen recursos tecnológicos adecuados para emplearlos como herramientas para generar conocimiento en mis alumnos?, etc. (Colorado-Aguilar B, Navarro R. E. 2012).

En el contexto de la sociedad de la información la web evolucionó de la 1.0 a la web 2.0, cambiando de lo estático a lo dinámico, ambiente que permite mayor trabajo educativo, destacando desde la perspectiva de García (2007:2) las siguientes ventajas: 
- Interactividad: Comunicación de manera bidireccional y multidireccional en entornos virtuales, permitiendo una mayor interrelación entre los interlocutores o usuarios del recurso tecnológico.

- Aprendizaje colaborativo: Comunidades de usuarios que favorecen el trabajo cooperativo y colaborativo permitiendo la interacción en la cual se logran aprendizajes con, entre y de otros a través de diferentes actividades de intercambio entre usuarios.

- Multidireccionalidad: Transmisión e intercambio de información que a través de diferentes aplicaciones de internet permite el tránsito simultáneo entre múltiples destinatarios.

- Libertad de edición y difusión: Permite la difusión, intercambio y colaboración en la edición de ideas y trabajos entre la multitud de usuarios de la red.

De esta forma, si bien existen sofisticados recursos tecnológicos que permiten un sinnúmero de aplicaciones, gran manejo de información y comunicación, es fundamental el enfoque que bajo criterios educativos puedan ser evaluados para ser utilizados como herramientas tecnológicas en el ámbito educativo, ya que "la facilidad que tiene un medio tecnológico para hacer accesible su aprendizaje, uso y aplicación en el desarrollo de habilidades cognitivas, informáticas y de comunicación le permitan al docente realizar su práctica educativa con mayor eficacia, eficiencia y satisfacción, siendo ésta última categoría la que facilita el aprendizaje”. (Cobo, 2005:125).

Si bien los criterios de usabilidad proporcionan un análisis del recurso para determinar si se adaptan o no a un contexto y uso específico, el enfoque pedagógico proporciona las bases fundamentales para enriquecer la utilización de herramientas tecnológicas en el proceso educativo.

Cabe destacar, que el proceso educativo no refiere una guía estructurada dado que no es un proceso que se ajuste plenamente a condiciones establecidas, es importante considerar factores que influyen directamente en esta labor, de acuerdo con Díaz (2010:3):

La práctica docente se encontrará fuertemente influida por la trayectoria de vida del profesor, el contexto socioeducativo donde se desenvuelva, el proyecto curricular en el que se ubique, las opciones pedagógicas que conozca o se lo exijan, así como las condiciones que tenga en la institución escolar. 


\section{La psicología de la educación y el análisis de las prácticas educativas que incorporan las TIC.}

El análisis de las prácticas educativas, es decir, los esfuerzos dirigidos a describir, comprender y explicar los factores y procesos implicados en la planificación y desarrollo de actividades de enseñanza y aprendizaje, exige la adopción de una mirada multidisciplinar que integre las aportaciones de las diferentes disciplinas y ámbitos de conocimiento. El hecho de que las prácticas educativas que son objeto de análisis incorporen recursos propios de las nuevas tecnologías de la información y la comunicación, no supone, para estos efectos, ninguna salvedad. Más bien al contrario, obliga en buena lógica a ampliar aún más, si cabe, el horizonte de la mirada, con el fin de incluir las aportaciones provenientes del análisis tecnológico en sus diferentes vertientes. (Coll C. 2001).

La valoración de la potencialidad de las TIC para el aprendizaje está estrechamente relacionada con las posibilidades que ofrecen para representar, procesar, transmitir y compartir información. Algunas de estas posibilidades, como la capacidad para transmitir cantidades ingentes de información de forma prácticamente instantánea a cualquier lugar, por alejado que se encuentre, o la facilidad de acceso a la misma, son evidentes. Pero ni información es sinónimo de conocimiento ni la recepción o el acceso a la información garantiza el aprendizaje. La información se convierte en conocimiento y el acceso a la información da lugar al aprendizaje cuando actuamos sobre ella, la procesamos, la organizamos, nos la apropiamos, la utilizamos y la confrontamos con otros; en suma, cuando somos capaces de darle significado y sentido. Además, hoy sabemos que estas operaciones que nos permiten transformar la información en conocimiento no pueden ni deben ser concebidas como operaciones estrictamente individuales. Aprendemos siempre de otros y con otros. El aprendizaje, especialmente el aprendizaje intencional, como es en buena medida el que tiene lugar en las situaciones educativas formales y escolares, es el resultado de complejos procesos interactivos y comunicativos. (Coll C. 2001).

\section{La educación a distancia. ¿Un nuevo paradigma de enseñanza y de aprendizaje?}

En los últimos años la educación a distancia tecnológica ha pasado en muy poco tiempo de ser considerada una modalidad educativa de "segunda fila" a ser muy valorada por todos los sistemas y niveles educativos. Esta alta apreciación actual ha originado tanto la aparición de foros 
especializados sobre la educación a distancia y sus implicaciones, como la extensión de ciertas discusiones sobre el tema en otros ámbitos educativos que tradicionalmente han impartido docencia en la educación presencial. (Barberá E, Badia A, Mominó J. M. 2001).

Desde perspectivas próximas a la psicología de la educación posiblemente no se apreciaría un cambio real en el paradigma que sustenta los procesos de enseñanza y de aprendizaje con soporte tecnológico. Incluso valorando la imprescindible contribución de las Tecnologías de la Información y la Comunicación (TIC) a la educación a distancia, se continúa propugnando que este hecho no resulta determinante para considerar que este tipo de educación, interpretado únicamente desde el punto de vista tecnológico y bajo sus criterios, necesita un tratamiento específico desde un punto de vista teórico. (Barberá E, Badia A, Mominó J. M. 2001).

Esto equivaldría a afirmar que las ideas clave que deberían fundamentar y orientar la valoración de los procesos de enseñanza y de aprendizaje, que se desarrollan en los entornos virtuales educativos, deben originarse desde posiciones teóricas similares a las que ayudan a interpretar la construcción de conocimiento que se lleva a cabo en entornos educativos presenciales. (Barberá E, Badia A, Mominó J. M. 2001).

Rivero Cárdenas I, Gómez Zermeño M, Abrego Tijerina R. (2013), evaluó, en su estudio la selección de estrategias didácticas que favorecen la práctica pedagógica a través del uso de las TIC. Los resultados muestran que las TIC se consideran un aliado tanto para la Institución, en cuanto al desarrollo de Proyectos de Tecnología Educativa a través de planes y programas, como para el docente siendo estas un valioso recurso que apoya el proceso de enseñanza-aprendizaje que ocurre dentro de las aulas.

Según lo expuesto en una investigación realizada por Colorado-Aguilar, Navarro (2012), la necesidad de contar con un fundamento teórico y didáctico de la usabilidad de TIC como parte de la formación inicial del docente, puntualizando que a través de la interacción docente-TIC se podrá avanzar en conocimiento y comprensión de los diferentes recursos digitales, factores que intervienen para dar al docente mayor seguridad en el uso continuo y la reflexión especializada de acuerdo con su área de conocimiento y poder identificar las TIC que por sus atributos contribuyan de manera significativa en su práctica educativa. Otro estudio el cual tuvo como finalidad conocer las percepciones de los jóvenes de un liceo municipal de la Región 
Metropolitana de Chile, acerca del uso de las tecnologías de información en el ámbito escolar. Los resultados apuntan a que los alumnos perciben que la tecnología es una necesidad inmediata, a la cual el liceo no está respondiendo adecuadamente, por lo que ellos recurren a estrategias alternativas de aprendizaje, que suplen dicha necesidad. (Peña I S et al. 2006).

Para dar por concluido nuestro estudio refleja una necesidad inminente que la formación inicial y continua de los profesores incorpore el desarrollo de habilidades, destrezas y conocimientos del uso de las tecnologías en el ámbito escolar, lo que se logrará siempre cuando los profesores den sentido a las tecnologías y al aporte que éstas tienen en el proceso de enseñanza/aprendizaje.

\section{Referencias bibliográficas}

Barberá E, Badia A, Mominó J. M. 2001. La incógnita de la Educación a Distancia. [Consulta 25 julio 2016]. Recuperado de http://revistas.um.es/redu/article/view/11511/11091

Coll C. (2001). Psicología de la educación y prácticas educativas mediadas por las tecnologías de la información y la comunicación. [Consulta 25 julio 2016]. Recuperado de http://www.redalyc.org/html/998/99815899016/

Colorado-Aguilar B, Navarro R. E. 2012. La usabilidad de TIC en la práctica educativa RED. Revista de Educación a Distancia. Número 30. [Consulta 17 mayo 2016]. Recuperado de http://www.um.es/ead/red/30

Cobo, C. (2005). Organización de la información y su impacto en la usabilidad de las tecnologías interactivas. Tesis doctoral de la Universidad Autónoma de Barcelona. En red. [Consulta 17 mayo 2016]. Recuperado en: http://www.tdx.cat/bitstream/handle/10803/4090/ccr1de1.pdf?sequence=1

Díaz, F. y Hernández, G. (2010). Estrategias docentes para un aprendizaje significativo.

Una interpretación constructivista. México: Mc Graw Hill.

García, L. (2007). ¿Web 2.0 vs web 1.0? Boletín Electrónico de noticias de Educación a distancia. En red. [Consulta 25 junio 2016]. Recuperado en:http://www.raco.cat/index.php/dim/article/viewFile/76637/98327 
Peña I S, Borrero A M, Marchant P, González G, Novoa D. (2006). Percepciones de jóvenes acerca del uso de las tecnologías de información en el ámbito escolar. Última década, 14(24), 37 60. [Consulta 17 mayo 2016]. Recuperado de https://dx.doi.org/10.4067/S071822362006000100003

Rivero Cárdenas I, Gómez Zermeño M, Abrego Tijerina R (2013). Tecnologías educativas y estrategias didácticas: criterios de selección. Revista Educación y Tecnología, №3.

Woolfolk A. (2010). Psicología educativa. Novena edición. Universidad del estado de Ohio. 